\title{
Intracellular events regulating cross-presentation
}

\section{Claudia S. Wagner ${ }^{\dagger}$, Jeffrey E. Grotzke ${ }^{\dagger}$ and Peter Cresswell*}

Department of Immunobiology, Yale University Medical Center, New Haven, CT, USA

Edited by:

Christian Kurts, Friedrich

Wilhelms-Universität Bonn, Germany

\section{Reviewed by:}

William Ross Heath, The University of Melbourne, Australia

Sammy Bedoui, The University of

Melbourne, Australia

Sven Burgdorf, Rheinische

Friedrich-Wilhelms-University,

Germany

\section{*Correspondence:}

Peter Cresswell, Department of Immunobiology, Yale University Medical Center, 300 Cedar Street, TAC S669, PO Box 208011, New Haven, CT, USA. e-mail:

peter.cresswell@yale.edu

${ }^{\dagger}$ Claudia S. Wagner and Jeffrey E. Grotzke have contributed equally to this work.
Cross-presentation plays a fundamental role in the induction of CD8-T cell immunity. However, although more than three decades have passed since its discovery, surprisingly little is known about the exact mechanisms involved. Here we give an overview of the components involved at different stages of this process. First, antigens must be internalized into the cross-presenting cell. The involvement of different receptors, method of antigen uptake, and nature of the antigen can influence intracellular trafficking and access to the cross-presentation pathway. Once antigens access the endocytic system, different requirements for endosomal/phagosomal processing arise, such as proteolysis and reduction of disulfide bonds. The majority of cross-presented peptides are generated by proteasomal degradation. Therefore, antigens must cross a membrane barrier in a manner analogous to the fate of misfolded proteins in the endoplasmic reticulum (ER) that are retrotranslocated into the cytosol for degradation. Indeed, some components of the ER-associated degradation machinery have been implicated in cross-presentation. Further complicating the matter, endosomal and phagosomal compartments have been suggested as alternative sites to the ER for loading of peptides on major histocompatibility complex class I molecules. Finally, the antigen presenting cells involved, particularly dendritic cell subsets and their state of maturation, influence the efficiency of cross-presentation.

Keywords: dendritic cell, MHC class I, endocytosis, phagocytosis

\section{CROSS-PRESENTATION: AN OVERVIEW}

In the broadest sense, cells contain two different antigen processing pathways that serve to present peptides to T lymphocytes. These pathways and the machinery required for them have distinct roles in the immune system and function to sample different environments for antigenic peptides. Major histocompatibility complex class I (MHC-I) molecules are loaded in the endoplasmic reticulum (ER) with peptides derived from degradation of cytosolic proteins by the proteasome, and these MHC-I/peptide complexes are then surface expressed and presented to $\mathrm{CD} 8^{+} \mathrm{T}$ cells. MHCII molecules exit the ER in association with the invariant chain, which occupies their peptide binding groove. In the endocytic pathway proteolysis results in the degradation of the invariant chain leaving the residual CLIP fragment in the binding groove. In a process catalyzed by HLA-DM (in humans) CLIP is replaced by peptides derived by proteolysis from proteins resident or internalized into the endocytic pathway. After surface expression, these are presented to $\mathrm{CD} 4^{+} \mathrm{T}$ cells. Hence, MHC-I generally serves as a reporter of intracellular infection, while MHC-II senses the antigens present in the extracellular milieu.

We now know that in professional antigen presenting cells (APC), peptides derived from exogenously acquired antigens can be presented on MHC-I. This process is known as crosspresentation. Furthermore, $\mathrm{CD} 8^{+} \mathrm{T}$ cells can be primed to such antigens by dendritic cells (DCs), a process termed cross-priming. The term was originally introduced to describe $\mathrm{CD} 8^{+} \mathrm{T}$ cell sensitization to minor histocompatibility antigens in transplantation situations (Bevan, 1976). The importance of cross-priming for the generation of $\mathrm{CD}^{+} \mathrm{T}$ cell-mediated immunity in general is a topic of debate, but cross-priming certainly plays an important role during priming of anti-tumor $\mathrm{CD} 8^{+} \mathrm{T}$ cells as well as priming the $\mathrm{CD}^{+}$response to pathogens which do not directly infect DCs.

Although various cell types, even including non-professional antigen-presenting cells like endothelial cells or modified 293T cells (Bagai et al., 2005; Giodini et al., 2009), are able to crosspresent under certain conditions, DCs are the most important cross-presenting cells in vivo (Jung et al., 2002). In mice, several DC subsets are competent for cross-presentation, with the lymphoidorgan resident CD8 $\alpha^{+}$DC (Heath et al., 2004; Hildner et al., 2008) and dermal migratory CD103 ${ }^{+}$DCs (Bedoui et al., 2009; Henri et al., 2010) defined as the main cross-presenting DCs. The search for human counterparts specialized in cross-presentation is ongoing, and considerable progress in characterization of human DC subsets has been made lately, including the discovery of a likely equivalent to the mouse CD8 $\alpha^{+}$DC subset (Villadangos and Shortman, 2010).

Cross-presentation of exogenous antigens raises interesting and important biochemical and cell biological questions. How do internalized proteins that are localized in the endocytic system gain access to the MHC-I processing and presentation machinery normally present in the ER? Over three decades have been spent defining how this process occurs. In a seminal study, Rock and colleagues demonstrated that presentation of exogenously derived antigens required proteasomal degradation, and therefore access to the cytosol (Kovacsovics-Bankowski and Rock, 1995). Consistent with this the ribosomal inhibitor protein gelonin was found to inhibit protein synthesis when added to cells, demonstrating that an intact protein can access the cytosol. An alternative mechanism, described by Harding and colleagues, involves endosomal 
processing of exogenous antigens and peptide binding to MHC-I in the endocytic system (Pfeifer et al., 1993). This is known as the vacuolar pathway. We now know that cross-presentation can occur through multiple pathways, including antigenic processing in the endocytic system and/or in the cytosol after translocation from endosomes or phagosomes. MHC-I binding after cytosolic processing may occur through several different mechanisms. After antigens reach the cytosol and are degraded by the proteasome, the resulting peptides can be transported into the ER or phagosomes via the transporter associated with antigen processing (TAP), where loading onto MHC-I can occur (Ackerman et al., 2003; Guermonprez et al., 2003; Houde et al., 2003; Grotzke et al., 2009). To complicate matters further, recent evidence suggests that peptide transport into phagosomes may involve an as yet unidentified, novel, transporter (Merzougui et al., 2011).

Although the broad pathways by which cross-presentation occurs have been elucidated, only now are more definitive molecular studies emerging. Moreover, many questions are still unanswered. Which cytosolic pathway is most widely used by cells? What role, if any, do endocytic receptors play in crosspresentation? How do antigens reach the cytosol? What factors help achieve the exquisite efficiency of this process that must occur in vivo? Are pathogens able to specifically inhibit cross-presentation? How is cross-presentation regulated in DCs? This review will focus on the known molecular mechanisms of cytosolic cross-presentation, while the other mechanisms will be covered by other authors. The second half of the review concentrates on the role of DC maturation in the context of cross-presentation.

\section{ANTIGEN UPTAKE BY PROFESSIONAL APC}

Early studies of cross-presentation demonstrated that the process is not simply a matter of extracellular processing and cell surface peptide loading (Pfeifer etal., 1993; KovacsovicsBankowski and Rock, 1995). For particulate antigens, phagocytosis is required for subsequent cross-presentation, and considerable evidence suggests that endocytosis is required for soluble antigens. The endocytic pathway ultimately leads to lysosomes and the phagocytic pathway progresses to phagolysosomes, but extensive proteolysis is incompatible with successful cross-presentation and a number of papers have implicated early endosomes and phagosomes in cross-presentation. A key question is whether different receptors deliver cargo preferentially to compartments that are capable of mediating cross-presentation. Several groups have shown that during and/or subsequent to phagocytosis, ER components are delivered to the phagosome (Ackerman et al., 2003, 2006; Guermonprez et al., 2003; Houde et al., 2003). A major focus has been on the role of this process in delivering the components necessary for translocation of antigenic proteins into the cytosol (see below), but in fact most or all of the components necessary for cross-presentation, including MHC-I and the peptide loading complex (PLC), as well as ER-derived retrotranslocation machinery, are recruited to the phagosome, creating a cross-presentation competent organelle. Whether delivery of ER components to the phagosome is required for cytosolic cross-presentation has been difficult to determine, but at least one study suggests that MyD88 signaling enhances delivery of ER components to endosomes and that this is required for cross-presentation of soluble ovalbumin (OVA; Burgdorf et al., 2008).

If phagocytosis of antigens results in the formation of a compartment that is competent for cross-presentation, is the ability to cross-present determined solely by the ability to phagocytose the antigen? It should be noted that an ER contribution to phagosomes occurs not only in DC, but also in cells that are much less efficient at cross-presentation, such as macrophages (Gagnon et al., 2002; Houde et al., 2003) and even a normally non-phagocytic cell line (Giodini et al., 2009). Furthermore, in Dictyostelium, not only are the ER proteins calnexin and calreticulin recruited to phagosomes, they appear to be required for phagocytosis (MullerTaubenberger et al., 2001). This suggests that ER-recruitment is an evolutionarily conserved process and not restricted to cells that can cross-present. Consistent with this, introduction of a phagocytic receptor (Fc $\gamma$ RIIa) into the non-cross-presenting cell line HEK293T leads to ER-recruitment to the phagosome and crosspresentation (Giodini et al., 2009). However, it is difficult to tease apart the role of receptor-specific effects and phagocytosis itself. Moreover, there is experimental evidence demonstrating that phagocytosis is not always sufficient to mediate cross-presentation, even in DC (Schnorrer et al., 2006).

Macrophages and DCs express a multitude of cell surface receptors that can mediate endocytosis and phagocytosis, but there is a dearth of data regarding the role that individual cell surface receptors and their associated intracellular trafficking and signaling pathways play in the process. Under certain conditions, specific receptors such as DEC-205 may induce cross-presentation more efficiently than other receptors such as DC-SIGN (Bozzacco et al., 2007), but the mechanisms that lead to this superiority have not been addressed. How could the internalizing receptor influence cross-presentation? First, certain receptors could traffic to a specific compartment that is highly competent for cross-presentation. Such a compartment may avoid the decreased $\mathrm{pH}$ and increased proteolysis detrimental for the process (Savina et al., 2006). Second, receptors could directly recruit additional components necessary for cross-presentation. Third, receptors and associated signaling adaptors could initiate a specialized signaling response that initiates cross-presentation. These receptor-specific responses could also serve to determine whether the cross-presented antigen promotes tolerance or an active CD8 response. The ability of a receptor to mediate cross-presentation and cross-priming in vivo is likely dependent on the antigen and its immunogenic potential, the delivery mechanism, co-stimulation, and the immune microenvironment.

The following receptors have been implicated in crosspresentation: Fc receptors (FcR), scavenger receptors (SR), DCSIGN (Dendritic Cell- $\underline{S} p e c i f i c$ ICAM-3 Grabbing NonIntegrin), MR (Mannose Receptor), DEC-205 (Dendritic and Epithelial Cells, $205 \mathrm{kDa}$ ), CLEC9A (C-type Lectin domain family 9A), DCIR (DC ImmunoReceptor), and LOX-1 (Lectin-like Oxidized Low-density Lipoprotein Receptor 1 ; Regnault et al., 1999; Bonifaz etal., 2002; Delneste etal., 2002; Berwin etal., 2003; Shakushiro et al., 2004; Tacken et al., 2005; Burgdorf et al., 2006; Bozzacco et al., 2007; Sancho et al., 2008, 2009; Klechevsky et al., 2010). In the case of MR, which internalizes OVA due to mannosylation, cross-presentation is dependent on co-ligation of 
TLR4 from contaminating endotoxin and subsequent MyD88 signaling (Burgdorf et al., 2008). Thus, internalization alone is not necessarily sufficient for cross-presentation, even by professional APC. Consistent with this, cross-presentation by CLEC9A, a receptor specifically expressed on the cross-presenting DC subset (Sancho et al., 2008), requires a hemITAM motif (containing only a single YxxL motif) in the cytoplasmic tail and subsequent syk signaling for efficient cross-presentation (Sancho et al., 2009). Notably, CLEC9A is required for recognition, but not uptake of its physiological ligand (necrotic cells). This suggests that cross-presentation may use one receptor for antigen uptake and a different receptor/adaptor protein to trigger the appropriate pathway in DCs.

The precise mechanisms by which receptor-induced signaling pathways initiate cross-presentation are poorly understood. In the case of CLEC9A-mediated cross-presentation, syk was found to associate with the hemITAM motif and be required for cross-presentation (Sancho et al., 2009). Similarly, cells deficient in FcR $\gamma$ or DAP12 adaptors, which signal through ITAM motifs, are deficient in cross-presentation of particulate antigens (Graham et al., 2007). Although the ITAM present in FcR has not been reported to be required for cross-presentation, FcR signaling is initiated by ITAM phosphorylation and syk recruitment (Swanson and Hoppe, 2004). These cases suggest that ITAM or hemITAM phosphorylation and subsequent syk signaling may be an important pathway. However, an antigen bound to MR can be cross-presented even though MR lacks an ITAM motif or known association with an ITAM-containing adaptor (Burgdorf et al., 2006). Similarly, the scavenger receptor SR-A1 can mediate cross-presentation while lacking an ITAM or known association of an ITAM-containing adaptor (C. Wagner and P. Cresswell, unpublished data), and mutation of the hemITAM motif in DC-SIGN does not affect cross-presentation of OVA bound to latex beads (C. Wagner and P. Cresswell, unpublished data). Finally, DCIR is able to cross-present targeted antigen even though it has an ITIM inhibitory motif (Klechevsky et al., 2010). These data suggest that there may be a general, unidentified signaling requirement for cross-presentation, that receptor-specific pathways can influence cross-presentation, and/or that incorporation of signals from multiple pathways may determine the fate of internalized antigens. Identification of receptors and associated signaling pathways that can activate cross-presentation should yield promising candidates to target for more efficient anti-tumor therapies and anti-pathogen vaccines.

To date, there is little to no evidence to show that individual phagocytic receptors impact the recruitment to the phagosome of additional proteins required for cross-presentation. In the case of MR-mediated cross-presentation, contaminating endotoxin is required for TLR4 ligation, MyD88 signaling, and recruitment of PLC members to endosomes (Burgdorf et al., 2008). Also, an ITAM signaling pathway not involved in uptake of bead- or bacteria-associated antigens was shown to be required for recruitment of NOX2 to phagosomes and efficient cross-presentation (Graham et al., 2007). These data suggest that recruitment of factors that enhance cross-presentation is determined not by the phagocytic receptor, but more likely by co-ligation of a second receptor.

\section{ANTIGEN UNFOLDING AND CYTOSOLIC TRANSLOCATION}

One of the more intriguing and perplexing questions about crosspresentation is how antigens access the cytosol. After initial studies showed that the proteasome is required for most examples of crosspresentation, with the underlying assumption that this reflects cytosolic proteolysis, how antigens cross the phagosomal membrane has been a major topic of study. A major leap forward was the finding that at least some of the machinery that functions in ER-associated degradation (ERAD), which translocates unfolded proteins from the ER lumen to the cytosol for degradation (Vembar and Brodsky, 2008), is localized to phagosomes in APCs and facilitates antigen translocation to the cytosol (Guermonprez et al., 2003; Houde et al., 2003; Imai et al., 2005; Ackerman et al., 2006). Recently it has been found that the ER-Golgi intermediate compartment (ERGIC) SNARE protein Sec22b is required for the process (Cebrian et al., 2011). When Sec22b function was inhibited using RNAi, DCs were much less efficient in translocating antigens to the cytosol. However, the components involved in antigen translocation to the cytosol and whether the nature of the antigen influences the process remain unclear. It is also unclear if there are specialized components of the retrotranslocation machinery in the phagosome and cytosol that function in cross-presentation but not in ERAD. Other open questions are whether there is a specific time window in which the retrotranslocation machinery is localized to phagosomes/endosomes, and whether the process is regulated by factors such as the maturation state of the cell or identity of the internalizing receptor. Also, as is the case with ERAD, the nature of the channel through which antigens are translocated remains a subject of debate.

Cells devote considerable energy to the production of secretory proteins. Not all of these proteins fold correctly and therefore need to be degraded before they are allowed to accumulate in the ER. Cells contain a structured complex of proteins that functions constitutively in the ER to facilitate degradation of misfolded proteins, i.e., ERAD. ERAD requires chaperones that recognize terminally misfolded proteins, a translocation channel, ubiquitination machinery, deglycosylating enzymes, and other accessory proteins (Vembar and Brodsky, 2008). In mammalian and yeast cells, the ERAD complex forms around a central E3 ubiquitin ligase, whose identity differs depending on the aberrant protein (Kostova et al., 2007). In mammalian cells, the most widely studied E3 ligases involved in ERAD are hrd1 and gp78, which function in the translocation of a number of ERAD substrates. Once a protein is identified as misfolded and targeted to the retrotranslocation channel it can be ubiquitinated during translocation, targeting it for degradation. After the substrate has partially entered the cytosol, the AAA ATPase VCP/p97 (cdc48 in yeast) generally functions to extract it into the cytosol (Ye et al., 2001; Braun et al., 2002; Jarosch etal., 2002; Rabinovich et al., 2002). The cytosolic chaperone hsp90 (Taylor et al., 2010), or even proteasomes themselves (Mayer et al., 1998; Lee et al., 2004; Lipson et al., 2008), have been proposed to mediate the dislocation event for substrates which are not dependent on p97. Overall, the function of ERAD is translocating misfolded proteins across a membrane and targeting them for proteasomal degradation before their accumulation results in induction of the unfolded protein response. 
The process of ERAD is very similar to antigen translocation into the cytosol for cross-presentation. Initial studies demonstrated that one potential retrotranslocation channel, sec61, is localized to phagosomes and that ubiquitinated proteins and proteasomes are associated with phagosomes (Guermonprez et al., 2003; Houde et al., 2003). Furthermore, ERAD components such as sec61, p97, and Bip can be co-immunoprecipitated with exogenously added OVA and vice versa (Imai et al., 2005). Together, these data suggest that the factors necessary for retrotranslocation are accessible to internalized antigens, even if the nature of the retrotranslocation channel(s) is unclear. The presence of functional retrotranslocation machinery has been confirmed by our laboratory and others. After internalization of latex beads and luciferase by a DC-like cell line and subsequent phagosome purification, translocation of phagosomal luciferase across the membrane requires addition of cytosol or recombinant p97 (Ackerman et al., 2006), demonstrating that p97 can function in phagosomal translocation as well as ERAD. Moreover, knockdown of $\mathrm{p} 97$ function by either expression of a dominant negative mutant or by siRNA inhibits cross-presentation (Imai et al., 2005; Ackerman et al., 2006). In addition to p97, hsp90 has also been implicated in both retrotranslocation and cross-presentation. Cells deficient in Hsp90 $\alpha$ by knockout, siRNA knockdown, or pharmacological inhibition show decreased cross-presentation of soluble or cell-associated OVA (Ichiyanagi et al., 2010). Hsp90 can contribute to antigen translocation to the cytosol (Imai et al., 2011) or to cytosolic refolding of proteins after translocation (Giodini and Cresswell, 2008).

Although ERAD components are recruited to phagosomes from the ER as described above, other mechanisms may contribute to recruitment of ERAD components in the case of endosomes. Mannose receptor ligation by OVA results in polyubiquitination of the receptor leading to increased recruitment of p97, OVA translocation to the cytosol, and cross-presentation (Zehner et al., 2011). Poly- but not mono-ubiquitination was required for the recruitment of p97 and OVA translocation to the cytosol, suggesting that receptor ubiquitination can serve to recruit cytosolic components required for antigen dislocation. As p97 can directly interact with members of the retrotranslocation complex (Zhong et al., 2004; Li et al., 2005; Schulze et al., 2005; Ye et al., 2005; Morreale et al., 2009), which must already be localized to endosomes for translocation to occur, it is unclear why receptor polyubiquitination is required for this recruitment. However, the retrotranslocation complexes present in phagosomes and endosomes are still ill-defined and this may be a mechanism governing the ability of certain receptors to mediate cross-presentation.

The retrotranslocation of exogenously added antigens has been difficult to study due to the lack of a good readout for their access to the cytosol. Studies have relied on the use of toxins such as gelonin or exotoxin A (exoA) from Pseudomonas aeruginosa (Kovacsovics-Bankowski and Rock, 1995; Ackerman et al., 2006; Giodini and Cresswell, 2008; Giodini et al., 2009), enzymes such as HRP (Gil-Torregrosa et al., 2004), cytochrome $c$ (Lin et al., 2008) or luciferase (Giodini and Cresswell, 2008), or pulsing large amounts of soluble OVA onto cells and examination of OVA in the cytosolic fraction (Burgdorf et al., 2008;
Imai et al., 2011). No assay system for tracing particulate antigens has been devised yet, and all of these methods using soluble antigens have their drawbacks. In the case of exoA, there is evidence demonstrating that exoA can inhibit retrotranslocation of radioactive-labeled peptides from microsomes (Koopmann et al., 2000). ExoA has also been shown to inhibit cross-presentation of soluble proteins and immune complexes as well as inhibit presentation of bacterially and parasite-derived antigens (Ackerman et al., 2006; Giodini etal., 2009; Goldszmid et al., 2009; Grotzke et al., 2009). However, the target of inhibition remains unknown. When pulsing enzymes or large amounts of protein antigen onto cells, a common problem is that protein or enzymatic activity found in the cytosol could be due to contamination during processing or lysosomal "bursting" and not true retrotranslocation. Until an assay is developed that directly measures retrotranslocation, results need to be interpreted with caution.

During ERAD, many substrates are unfolded before translocation to the cytosol. If cross-presentation utilizes the same retrotranslocation machinery as ERAD, then cross-presented antigen should also be unfolded before translocation. Indeed, several reports have shown a requirement for acidification or partial lysosomal proteolysis of antigens that are cross-presented in a proteasome-dependent manner (Fonteneau et al., 2003; Giodini et al., 2009), suggesting that processing of the internalized protein or at least breakdown of immune complexes or apoptotic cells are required prior to translocation. Chaperones such as calnexin, calreticulin, Bip, and the ER enzyme protein disulfide isomerase (PDI), have all been shown to localize to phagosomes and may play a role in protein unfolding (Ackerman et al., 2003; Guermonprez et al., 2003; Houde et al., 2003; Imai et al., 2005). For the case of ERAD substrates containing disulfide bonds, several studies demonstrate that reduction is required for, or enhances, retrotranslocation (Molinari et al., 2002; Dong et al., 2008; Ushioda et al., 2008). Similarly, for cross-presented antigens with disulfide bonds, it is likely that reduction and unfolding is required for cytosolic access. The only known thiol reductase present in phagosomes and lysosomes prior to ER acquisition is $\gamma$-interferon inducible lysosomal thiol reductase (GILT). GILT-deficient DC are deficient in their ability to cross-present gB, a HSV-1 glycoprotein that contains five disulfide bonds, but showed no defect in crosspresentation of a protein that did not contain disulfide bonds (Singh and Cresswell, 2010). Furthermore, GILT-deficient mice showed a decrease in cross-priming anti-gB and anti-influenzaspecific $\mathrm{CD}^{+} \mathrm{T}$ cells. These results underscore the importance of protein unfolding for cross-presentation, and demonstrate that further characterization of the mechanisms that aid protein unfolding while at the same time limiting lysosomal proteolysis are needed.

\section{CROSS-PRESENTATION IN THE CONTEXT OF DC MATURATION}

The ability to cross-present not only differs between various cell types or DC subsets, but the maturation state of the crosspresenting DCs also plays an important role. Microbial products, inflammatory cytokines or mediators of tissue damage induce a process of maturation in DCs that, besides changes in phenotype and motility, also involves changes in handling and presentation 
of antigens. These modifications significantly affect MHC-IIrestricted presentation, but also influence cross-presentation. Maturation is a potentially attractive approach to dissecting the molecular mechanisms regulating cross-presentation. However, care needs to be taken to separate effects on $\mathrm{T}$ cells resulting from enhanced co-stimulation from a true effect on the formation of MHC-I/peptide complexes.

Depending on the timing there are different scenarios for how DC maturation may affect cross-presentation. One can ask how DC activation affects cross-presentation of antigens that are acquired together with or shortly prior to the maturation event, such as antigens acquired from virally infected cells. Experimentally this may be represented by the administration of a defined antigen followed by or combined with specific ligand for innate immune receptors, such as Toll-like receptors (TLRs). Alternatively one can ask if DCs are still able to cross-present antigens that they encounter in an already matured state. This would be important in a situation in which an individual encounters a pathogen while already undergoing a response to prior infection. We will discuss the latter situation first.

\section{CROSS-PRESENTATION IN MATURE DCS}

A rationale for cross-presentation being controlled through maturation comes from analogy to presentation on MHC-II. Both cross-presentation and MHC-II presentation serve to prime $\mathrm{T}$ cell responses, with antigens presented by either MHC-I to CD8 ${ }^{+} \mathrm{T}$ cells or to $\mathrm{CD}^{+}{ }^{+} \mathrm{T}$ cells via MHC-II. MHC-II-restricted antigen presentation is tightly regulated during maturation at several levels, including alterations in endocytic proteolysis, re-distribution of peptide loaded MHC-II molecules to the cell surface, enhanced stability of surface complexes and reduction of MHC-II biosynthesis (Wilson and Villadangos, 2005). With few exceptions (Drutman and Trombetta, 2010; Platt et al., 2010), these maturation-induced changes generally prevent MHC-II presentation of antigens by mature DCs. The proposed benefit is preservation of the MHC-II/ peptide complexes derived from antigens acquired at the onset of maturation (Villadangos et al., 2005). A pathogen-derived antigen that is acquired in the periphery by immature DCs will still be presented by mature DCs that have migrated to lymph nodes to prime $\mathrm{CD} 4^{+} \mathrm{T}$ cells.

For cross-presentation, the situation is more complex. There is a considerable overlap with pathways used simultaneously for endogenous MHC-I presentation, a process that is still operational in mature DCs (Gil-Torregrosa et al., 2004; Wilson et al., 2006). Supporting this notion, MHC-I synthesis and trafficking is not subjected to the same control during maturation as MHC-II. MHC-I synthesis is increased during maturation (Cella et al., 1997; Rescigno et al., 1998) and stability is not affected (Cella et al., 1997; Delamarre et al., 2003) or is only moderately affected (Ackerman and Cresswell, 2003). One could argue that crosspresentation should still be operational in mature DCs to allow initiation of responses toward secondary pathogens, although the relatively short lifespan of matured DCs and the constant renewal by fresh immature DCs could take care of that problem (Kamath et al., 2002). If cross-presentation is indeed regulated by maturation in order to focus or preserve certain antigens, what could be the mechanism? Exogenous material taken up at the onset of maturation may be retained intracellularly until the mature DC reaches the lymph node, creating a form of antigenic memory. In mouse DC-like cell lines and to some extent in bone marrow DCs, storage of soluble (Lutz et al., 1997) or immune complexed antigens (van Montfoort et al., 2009) in distinct compartments with reduced proteolytic activity has been observed, either in immature DCs (Lutz et al., 1997) or in mature DCs (van Montfoort et al., 2009). Whether specialized storage compartments for exogenous antigens exist in all cross-presenting DCs is unknown. Also unknown is how antigen deposition and release are regulated. In a human DC-like cell line trafficking of MHC-I molecules to the cell surface was delayed in an immature state, suggesting that intracellular retention of preformed MHC-I/peptide complexes may occur (Ackerman and Cresswell, 2003).

Regardless of theoretical considerations, published evidence does not clearly argue for or against regulation of crosspresentation during maturation. As often, the truth may lie somewhere in between: cross-presentation in mature DCs may be compromised under certain circumstances, depending on the maturation stimulus and the nature or the form of the antigen that is encountered. With a few exceptions, studies have employed the OVA antigen to study the effect of maturation on crosspresentation. It should be noted that bone marrow-derived DCs require an additional maturation stimulus during or shortly after antigen uptake to cross-present soluble OVA (Delamarre et al., 2003; Gil-Torregrosa et al., 2004). Depending on the study, pretreatment of DCs with certain TLR ligands such as CpG, LPS, or poly (I:C), either did not alter or enhanced cross-presentation of subsequently acquired antigens (Regnault et al., 1999; Machy et al., 2000; Datta et al., 2003; Henri et al., 2007; Weck et al., 2007; Drutman and Trombetta, 2010; Platt et al., 2010). On the other hand, the ability to cross-present can also be lost after contact with TLR ligands, both after administration in vivo (Wilson et al., 2006) as well as after prolonged treatment of DCs in vitro (Gil-Torregrosa et al., 2004; Weck et al., 2007). We found that peptidoglycan, a common impurity in LPS preparations, can inhibit cross-presentation of viral antigens via signaling through cytoplasmic NOD receptors (Wagner and Cresswell, 2012).

Decreased antigen uptake (Gil-Torregrosa et al., 2004; Wilson et al., 2006; Weck et al., 2007) and a lack of transfer of antigen to the cytosol (Gil-Torregrosa et al., 2004) have been proposed to explain reduced cross-presentation by mature DCs. Decreased uptake is likely a contributing factor but cannot be the sole explanation, as mature DCs still take up considerable amounts of antigens, even if reduced compare to immature DCs (Datta et al., 2003; Drutman and Trombetta, 2010; Platt et al., 2010; Wagner and Cresswell, 2012; C. Wagner and P. Cresswell, unpublished data). In vivo, antigen availability is certainly also a regulatory factor. For example, immature DCs residing in the periphery may have access to pathogen-derived antigens at a local site of infection, unlike mature DCs that have already migrated to the lymph node and never come in contact with the new antigen. Regarding inhibition of antigen transfer to the cytosol, it would be very interesting to understand exactly how this process is impaired in mature DCs. Are the antigens routed to different compartments that lack components of the still unidentified transport machinery? Are the 
antigens degraded within endosomal/lysosomal compartments before they can be retrotranslocated? These are potential levels of regulation that are specific for cross-presentation and would not interfere with endogenous MHC-I peptide loading and presentation.

\section{PHAGOSOME MATURATION AND ANTIGEN DEGRADATION}

Major contributions to our understanding of cross-presentation came from a series of studies on the regulation of proteolysis and $\mathrm{pH}$ in DCs. DCs have a lower content of lysosomal proteases compared to macrophages (Delamarre etal., 2005) and the kinetics of acquisition of distinct proteases during phagosome maturation are slower in DCs than macrophages (LennonDumenil et al., 2002). Amigorena and co-workers demonstrated that, unlike macrophages, DCs limit acidification in phagosomes and inhibit proteolysis, thus promoting cross-presentation (Savina et al., 2006). DCs maintain $\mathrm{pH}$ levels above $\mathrm{pH} 7$ for several hours post-phagocytosis (Savina et al., 2006), in contrast to macrophages where the $\mathrm{pH}$ drops to $\mathrm{pH} 5$ within 15 min (Yates et al., 2005). The mechanism involves recruitment of the NADPH oxidase NOX2 to the phagosome, which drives alkalinization of the phagosomal lumen and is recruited in a Rab27-dependent fashion (Savina et al., 2006; Jancic et al., 2007). In CD8 $\alpha^{+}$DC, the GTPase Rac2 is responsible for assembly of the NOX 2 complex on phagosomes, while Rac1 directs NOX2 to the plasma membrane in CD8 $\alpha^{-}$ DC (Savina et al., 2009). A high pH would serve to limit proteolysis and thus favor cross-presentation. An elevated $\mathrm{pH}$ might also contribute in other ways to successful cross-presentation, such as influencing the conformation of proteins associated with translocation of antigens or proper assembly of the PLC. Although limited acidification may facilitate cross-presentation, a certain degree of proteolysis is necessary for pre-processing particulate antigens before translocation into the cytosol or for complete processing of antigens in case of the vacuolar pathway of crosspresentation. DCs may selectively use proteases active at a higher $\mathrm{pH}$ than most lysosomal proteases. One example is cathepsin S (Kirschke et al., 1989) which is enriched in DCs (Lennon-Dumenil et al., 2002) and has been shown to be involved in processing of antigens for the vacuolar pathway of cross-presentation (Shen et al., 2004).

Dendritic cell activation has been demonstrated to modify phagosome maturation and proteolysis. The assembly of the vacuolar proton pump in lysosomes is enhanced after activation with LPS, resulting in enhanced acidification and higher protein degradation (Trombetta et al., 2003), while phagocytosis induction combined with LPS treatment of DCs was found to delay acquisition of active proteases by phagosomes (LennonDumenil etal., 2002). This could mean that despite an overall enhancement of lysosomal activity after TLR4 triggering, proteolytic activity in phagosomes is actually reduced, potentially preserving antigens for cross-presentation. However, the latter study was based on a method using internalized beads coated with probes specific for cysteine proteases, and LPS-treated DC tend to be "sticky" and immobilize beads at the cell surface. Surface bound beads could potentially lead to an overestimation of initial uptake compared to untreated DC and also result in a mixed population of phagosomes, due to slow internalization of the surface bound beads over time (C. Wagner and P. Cresswell, unpublished data). Two further reports link NOX2 activity to TLR signaling and efficient intracellular bacterial killing: in human DC, NOX2 activity was increased in TLR ligand matured DCs (Vulcano et al., 2004), and in macrophages, NOX2 assembly was regulated by MyD88, a central adaptor protein for TLR signaling (Laroux et al., 2005). Thus, TLR signaling appears to regulate cross-presentation by modulating NOX2 activity and phagosomal $\mathrm{pH}$.

\section{CROSS-PRESENTATION OF ANTIGENS COMBINED WITH MATURATION STIMULI}

How does maturation that occurs simultaneously with or after antigen uptake relate to the ability to cross-present? $\mathrm{CD} 8 \alpha^{-}$cells, which are inferior to CD $8 \alpha^{+}$DCs in terms of cross-presentation, can be activated through $\mathrm{Fc} \gamma \mathrm{R}$ triggering and become competent for cross-presentation of immune complexes (den Haan and Bevan, 2002). Besides immune complexes (Regnault et al., 1999; den Haan and Bevan, 2002), cross-presentation can be induced by certain stimuli such as LPS (Gil-Torregrosa et al., 2004; West et al., 2004), disruption of cell contacts, or CD40L stimulation (Delamarre et al., 2003), but not by CpG (Datta et al., 2003; Delamarre et al., 2003), low-dose LPS, Poly (I:C), or TNF $\alpha$ (Delamarre et al., 2003). This means that OVA cross-presentation is induced by only a subset of maturation conditions that stimulate MHC-II presentation. Cross-presentation of physiological relevant antigens, such as viral proteins from infected cells, may have different requirements for maturation stimuli. These antigens are already delivered in a complex mix of activating signals, such as pathogen-derived TLR ligands and signals from dying cells. Under experimental settings, no additional external maturation stimuli are needed to induce cross-presentation.

What mechanisms are responsible for maturation-induced changes in cross-presentation? Early after activation, DCs transiently sequester endogenous ubiquitinated proteins in cytosolic aggregates, termed DALIS by Pierre and colleagues, a phenomenon proposed to favor the processing of internalized exogenous antigens for cross-presentation (Lelouard et al., 2004). It has been proposed that cross-presentation involves early endosomal compartments (Burgdorf etal., 2008; Di Pucchio et al., 2008; Belizaire and Unanue, 2009). During maturation, changes in phagosomal/endosomal routing could potentially also alter the fate of antigens. Blander and Medzhitov (2006b) proposed that the presence of a TLR ligand with an antigen in a phagosome favors MHC-II processing. Only antigens from phagosomes with TLR triggering are efficiently routed to lysosomes where invariant chain processing occurs. This offers a solution to the problem how an antigen-presenting cell would ensure that only harmful antigens and not phagocytosed selfantigens are presented to $\mathrm{T}$ cells. For cross-presentation, one could speculate that the effect would be the opposite, i.e., that TLR-dependent shuffling toward lysosomal degradation would impair efficient cross-presentation. It has also been reported that TLR signaling influences phagosome maturation in macrophages (Blander and Medzhitov, 2004). However, Russell and colleague were unable to detect TLR2 or TLR4-dependent regulation of phagosome maturation (Yates and Russell, 2005). Potential 
explanations for the discrepancy have been discussed by the authors (Blander and Medzhitov, 2006a; Russell and Yates, 2007). Another study showed that TLR stimulation recruits components of the autophagy pathway to phagosomes, resulting in enhanced phagosome maturation (Sanjuan et al., 2007). The latter three studies on TLR-dependent phagosome maturation have used macrophages and not all findings may apply to DCs.

Finally, maturation signals alone do not determine the crosspresentation ability of a DC. There are many subsets of peripheral DCs that mature after encounter of antigen and migrate to lymph nodes, yet only a specialized subset, defined by the expression of $\mathrm{CD} 103^{+}$(Bedoui et al., 2009; Henri et al., 2010) can cross-present with high efficiency. A particular transcriptional profile is likely to be responsible for this, because migratory $\mathrm{CD} 103^{+}$DCs are very similar to cross-presenting lymph node-resident $\mathrm{CD} 8 \mathrm{a}^{+}$DCs, sharing the marker XCR1 (Crozat et al., 2011).

\section{REFERENCES}

Ackerman, A. L., and Cresswell, P. (2003). Regulation of MHC class I transport in human dendritic cells and the dendritic-like cell line KG-1. J. Immunol. 170, 4178-4188.

Ackerman, A. L., Giodini, A., and Cresswell, P. (2006). A role for the endoplasmic reticulum protein retrotranslocation machinery during crosspresentation by dendritic cells. Immunity 25, 607-617.

Ackerman, A. L., Kyritsis, C., Tampe, R., and Cresswell, P. (2003). Early phagosomes in dendritic cells form a cellular compartment sufficient for cross presentation of exogenous antigens. Proc. Natl. Acad. Sci. U.S.A. 100, 12889-12894.

Bagai, R., Valujskikh, A., Canaday, D. H., Bailey, E., Lalli, P. N., Harding, C. V., and Heeger, P. S. (2005). Mouse endothelial cells cross-present lymphocyte-derived antigen on class I MHC via a TAP1- and proteasomedependent pathway. J. Immunol. 174, 7711-7715.

Bedoui, S., Whitney, P. G., Waithman, J., Eidsmo, L., Wakim, L., Caminschi, I., Allan, R. S., Wojtasiak, M., Shortman, K., Carbone, F. R., Brooks, A. G., and Heath, W. R. (2009). Crosspresentation of viral and self antigens by skin-derived $\mathrm{CD}_{103^{+}}$dendritic cells. Nat. Immunol. 10, 488-495.

Belizaire, R., and Unanue, E. R. (2009). Targeting proteins to distinct subcellular compartments reveals unique requirements for MHC class I and II presentation. Proc. Natl. Acad. Sci. U.S.A.106, 17463-17468.

Berwin, B., Hart, J. P., Rice, S., Gass, C., Pizzo, S. V., Post, S. R., and Nicchitta, C. V. (2003). Scavenger receptor-A mediates gp96/GRP94 and calreticulin internalization by antigen-presenting cells. EMBO J. 22, 6127-6136.

Bevan, M. J. (1976). Cross-priming for a secondary cytotoxic response to minor $\mathrm{H}$ antigens with $\mathrm{H}-2$ congenic cells which do not cross-react in the cytotoxic assay. J. Exp. Med. 143, 1283-1288.

Blander, J. M., and Medzhitov, R. (2004). Regulation of phagosome maturation by signals from Toll-like receptors. Science 304, 1014-1018.

Blander, J. M., and Medzhitov, R. (2006a). On regulation of phagosome maturation and antigen presentation. Nat. Immunol. 7, 1029-1035.

Blander, J. M., and Medzhitov, R. (2006b). Toll-dependent selection of microbial antigens for presentation by dendritic cells. Nature 440 , 808-812.

Bonifaz, L., Bonnyay, D., Mahnke, K., Rivera, M., Nussenzweig, M. C., and Steinman, R. M. (2002). Efficient targeting of protein antigen to the dendritic cell receptor DEC-205 in the steady state leads to antigen presentation on major histocompatibility complex class I products and peripheral $\mathrm{CD}^{+} \mathrm{T}$ cell tolerance. J. Exp. Med. 196, 1627-1638.

Bozzacco, L., Trumpfheller, C., Siegal, F. P., Mehandru, S., Markowitz, M., Carrington, M., Nussenzweig, M. C., Piperno, A. G., and Steinman, R. M. (2007). DEC-205 receptor on dendritic cells mediates presentation of HIV gag protein to $\mathrm{CD}^{+} \mathrm{T}$ cells in a spectrum of human MHC I haplotypes. Proc. Natl. Acad. Sci. U.S.A. 104, 1289-1294.

Braun, S., Matuschewski, K., Rape, M., Thoms, S., and Jentsch, S. (2002). Role of the ubiquitin-selective

\section{CONCLUDING REMARKS}

Understanding how maturation affects cross-presentation in vitro and in vivo is important for vaccination strategies and other immunotherapies, where the induction of maturation is a prerequisite for eliciting an effective $\mathrm{T}$ cell response. In addition, dissecting which factors influence the ability to cross-present during maturation will advance our understanding of the molecular process of cross-presentation. Studying maturation in vitro allows one to work with one cell type under defined conditions, where the modification of a single parameter, such as the addition of a TLR ligand, changes the outcome of cross-presentation. However, any advances obtained from in vitro systems need to be verified in more complex in vivo settings, a step where knockout animals have proven to be an invaluable tool. Models using infectious agents that can subvert antigen processing pathways will also contribute to our understanding. Last but not least, having the necessary reagents and readouts to follow antigens other than OVA is a major requirement for further progress.

CDC48(UFD1/NPL4) chaperone (segregase) in ERAD of OLE1 and other substrates. EMBO J. 21, 615-621.

Burgdorf, S., Lukacs-Kornek, V., and Kurts, C. (2006). The mannose receptor mediates uptake of soluble but not of cell-associated antigen for cross-presentation. J. Immunol. 176, 6770-6776.

Burgdorf, S., Scholz, C., Kautz, A., Tampe, R., and Kurts, C. (2008). Spatial and mechanistic separation of cross-presentation and endogenous antigen presentation. Nat. Immunol. 9, 558-566.

Cebrian, I., Visentin, G., Blanchard, N., Jouve, M., Bobard, A., Moita, C. Enninga, J., Moita, L. F., Amigorena, S., and Savina, A. (2011). Sec22b regulates phagosomal maturation and antigen crosspresentation by dendritic cells. Cell 147, 1355-1368.

Cella, M., Engering, A., Pinet, V., Pieters, J., and Lanzavecchia, A. (1997). Inflammatory stimuli induce accumulation of MHC class II complexes on dendritic cells. Nature 388, 782-787.

Crozat, K., Tamoutounour, S., Vu Manh, T. P., Fossum, E., Luche, H., Ardouin, L., Guilliams, M., Azukizawa, H., Bogen, B., Malissen, B., Henri, S., and Dalod, M. (2011). Cutting edge: expression of XCR1 defines mouse lymphoid-tissue resident and migratory dendritic cells of the CD8alpha ${ }^{+}$type. J. Immunol. 187, 4411-4415.

Datta, S. K., Redecke, V., Prilliman, K. R., Takabayashi, K., Corr, M., Tallant, T., Didonato, J., Dziarski, R., Akira, S., Schoenberger, S. P., and Raz, E. (2003). A subset of Toll-like receptor ligands induces cross-presentation by bone marrow-derived dendritic cells. J. Immunol. 170, 4102-4110.

Delamarre, L., Holcombe, H., and Mellman, I. (2003). Presentation of exogenous antigens on major histocompatibility complex (MHC) class I and MHC class II molecules is differentially regulated during dendritic cell maturation. J. Exp. Med. 198, 111-122.

Delamarre, L., Pack, M., Chang, H., Mellman, I., and Trombetta, E. S. (2005). Differential lysosomal proteolysis in antigen-presenting cells determines antigen fate. Science 307 , 1630-1634.

Delneste, Y., Magistrelli, G., Gauchat, J., Haeuw, J., Aubry, J., Nakamura, K., Kawakami-Honda, N., Goetsch, L., Sawamura, T., Bonnefoy, J., and Jeannin, P. (2002). Involvement of LOX-1 in dendritic cell-mediated antigen cross-presentation. Immunity 17, 353-362.

den Haan, J. M., and Bevan, M. J. (2002). Constitutive versus activationdependent cross-presentation of immune complexes by $\mathrm{CD} 8(+)$ and $\mathrm{CD} 8(-)$ dendritic cells in vivo. $J$. Exp. Med. 196, 817-827.

Di Pucchio, T., Chatterjee, B., SmedSorensen, A., Clayton, S., Palazzo, A., Montes, M., Xue, Y., Mellman, I., Banchereau, J., and Connolly, J. E. (2008). Direct proteasomeindependent cross-presentation of viral antigen by plasmacytoid dendritic cells on major histocompatibility complex class I. Nat. Immunol. 9, 551-557.

Dong, M., Bridges, J. P., Apsley, K., Xu, Y., and Weaver, T. E. (2008). ERdj4 and ERdj5 are required for endoplasmic reticulum-associated protein degradation of misfolded surfactant 
protein C. Mol. Biol. Cell 19, 26202630.

Drutman, S. B., and Trombetta, E. S. (2010). Dendritic cells continue to capture and present antigens after maturation in vivo. J. Immunol. 185, 2140-2146.

Fonteneau, J. F., Kavanagh, D. G., Lirvall, M., Sanders, C., Cover, T. L., Bhardwaj, N., and Larsson, M. (2003). Characterization of the MHC class I cross-presentation pathway for cell-associated antigens by human dendritic cells. Blood 102, 4448-4455.

Gagnon, E., Duclos, S., Rondeau, C., Chevet, E., Cameron, P. H., SteeleMortimer, O., Paiement, J., Bergeron, J. J., and Desjardins, M. (2002). Endoplasmic reticulum-mediated phagocytosis is a mechanism of entry into macrophages. Cell 110, 119-131.

Gil-Torregrosa, B. C., Lennon-Dumenil, A. M., Kessler, B., Guermonprez, P., Ploegh, H. L., Fruci, D., Van Endert, P., and Amigorena, S. (2004). Control of cross-presentation during dendritic cell maturation. Eur. J. Immunol. 34, 398-407.

Giodini, A., and Cresswell, P. (2008). Hsp90-mediated cytosolic refolding of exogenous proteins internalized by dendritic cells. EMBO J. 27, 201-211.

Giodini, A., Rahner, C., and Cresswell, P. (2009). Receptor-mediated phagocytosis elicits cross-presentation in nonprofessional antigen-presenting cells. Proc. Natl. Acad. Sci. U.S.A. 106, 3324-3329.

Goldszmid, R. S., Coppens, I., Lev, A., Caspar, P., Mellman, I., and Sher, A. (2009). Host ERparasitophorous vacuole interaction provides a route of entry for antigen cross-presentation in Toxoplasma gondii-infected dendritic cells. J. Exp. Med. 206, 399-410.

Graham, D. B., Stephenson, L. M., Lam, S. K., Brim, K., Lee, H. M., Bautista, J., Gilfillan, S., Akilesh, S., Fujikawa, K., and Swat, W. (2007). An ITAM-signaling pathway controls cross-presentation of particulate but not soluble antigens in dendritic cells. J. Exp. Med. 204, 2889-2897.

Grotzke, J. E., Harriff, M. J., Siler, A. C., Nolt, D., Delepine, J., Lewinsohn, D. A., and Lewinsohn, D. M. (2009). The Mycobacterium tuberculosis phagosome is a HLA-I processing competent organelle. PLoS Pathog. 5, e1000374. doi: 10.1371/ journal.ppat.1000374

Guermonprez, P., Saveanu, L., Kleijmeer, M., Davoust, J., Van Endert, P., and Amigorena, S. (2003). ER-phagosome fusion defines an MHC class I cross-presentation compartment in dendritic cells. Nature 425, 397-402.

Heath, W. R., Belz, G. T., Behrens, G. M., Smith, C. M., Forehan, S. P., Parish, I. A., Davey, G. M., Wilson, N. S., Carbone, F. R., and Villadangos, J. A. (2004). Crosspresentation, dendritic cell subsets, and the generation of immunity to cellular antigens. Immunol. Rev. 199, 9-26.

Henri, S., Poulin, L. F., Tamoutounour, S., Ardouin, L., Guilliams, M., De Bovis, B., Devilard, E., Viret, C., Azukizawa, H., Kissenpfennig, A., and Malissen, B. (2010). CD207+ CD103+ dermal dendritic cells crosspresent keratinocyte-derived antigens irrespective of the presence of Langerhans cells. J. Exp. Med. 207, 189-206.

Henri, S., Siret, C., Machy, P., Kissenpfennig, A., Malissen, B., and Leserman, L. (2007). Mature DC from skin and skin-draining $\mathrm{LN}$ retain the ability to acquire and efficiently present targeted antigen. Eur. J. Immunol. 37, 1184-1193.

Hildner, K., Edelson, B. T., Purtha, W. E., Diamond, M., Matsushita, H., Kohyama, M., Calderon, B., Schraml, B. U., Unanue, E. R., Diamond, M. S., Schreiber, R. D., Murphy, T. L., and Murphy, K. M. (2008). Batf3 deficiency reveals a critical role for CD8alpha+ dendritic cells in cytotoxic T cell immunity. Science 322 , 1097-1100.

Houde, M., Bertholet, S., Gagnon, E., Brunet, S., Goyette, G., Laplante, A., Princiotta, M. F., Thibault, P., Sacks, D., and Desjardins, M. (2003). Phagosomes are competent organelles for antigen crosspresentation. Nature 425, 402-406.

Ichiyanagi, T., Imai, T., Kajiwara, C., Mizukami, S., Nakai, A., Nakayama, T., and Udono, H. (2010). Essential role of endogenous heat shock protein 90 of dendritic cells in antigen cross-presentation. J. Immunol. 185, 2693-2700.

Imai, J., Hasegawa, H., Maruya, M., Koyasu, S., and Yahara, I. (2005). Exogenous antigens are processed through the endoplasmic reticulumassociated degradation (ERAD) in cross-presentation by dendritic cells. Int. Immunol. 17, 45-53.

Imai, T., Kato, Y., Kajiwara, C., Mizukami, S., Ishige, I., Ichiyanagi, T., Hikida, M., Wang, J. Y., and Udono, H. (2011). Heat shock protein 90 (HSP90) contributes to cytosolic translocation of extracellular antigen for cross-presentation by dendritic cells. Proc. Natl. Acad. Sci. U.S.A. 108, 16363-16368.
Jancic, C., Savina, A., Wasmeier, C., Tolmachova, T., El-Benna, J., Dang, P. M., Pascolo, S., Gougerot-Pocidalo, M. A., Raposo, G., Seabra, M. C., and Amigorena, S. (2007). Rab27a regulates phagosomal $\mathrm{pH}$ and NADPH oxidase recruitment to dendritic cell phagosomes. Nat. Cell Biol. 9, 367-378.

Jarosch, E., Taxis, C., Volkwein, C., Bordallo, J., Finley, D., Wolf, D. H., and Sommer, T. (2002). Protein dislocation from the ER requires polyubiquitination and the AAA-ATPase Cdc48. Nat. Cell Biol. 4, 134-139.

Jung, S., Unutmaz, D., Wong, P., Sano, G., De Los Santos, K., Sparwasser, T., Wu, S., Vuthoori, S., Ko, K., Zavala, F., Pamer, E. G., Littman, D. R., and Lang, R. A. (2002). In vivo depletion of CD11c+ dendritic cells abrogates priming of CD8 $+\mathrm{T}$ cells by exogenous cell-associated antigens. Immunity 17, 211-220.

Kamath, A. T., Henri, S., Battye, F., Tough, D. F., and Shortman, K. (2002). Developmental kinetics and lifespan of dendritic cells in mouse lymphoid organs. Blood 100, 1734-1741.

Kirschke, H., Wiederanders, B., Bromme, D., and Rinne, A. (1989). Cathepsin S from bovine spleen. Purification, distribution, intracellular localization and action on proteins. Biochem. J. 264, 467-473.

Klechevsky, E., Flamar, A. L., Cao, Y., Blanck, J. P., Liu, M., O’Bar, A., Agouna-Deciat, O., Klucar, P. Thompson-Snipes, L., Zurawski, S., Reiter, Y., Palucka, A. K., Zurawski, G., and Banchereau, J. (2010). Cross-priming CD8 $+\mathrm{T}$ cells by targeting antigens to human dendritic cells through DCIR. Blood 116, 1685-1697.

Koopmann, J. O., Albring, J., Huter, E., Bulbuc, N., Spee, P., Neefjes, J., Hammerling, G. J., and Momburg, F. (2000). Export of antigenic peptides from the endoplasmic reticulum intersects with retrograde protein translocation through the Sec61p channel. Immunity 13, 117-127.

Kostova, Z., Tsai, Y. C., and Weissman, A. M. (2007). Ubiquitin ligases, critical mediators of endoplasmic reticulum-associated degradation. Semin. Cell Dev. Biol. 18, 770-779.

Kovacsovics-Bankowski, M., and Rock, K. L. (1995). A phagosome-to-cytosol pathway for exogenous antigens presented on MHC class I molecules. Science 267, 243-246.

Laroux, F. S., Romero, X., Wetzler, L., Engel, P., and Terhorst, C. (2005). Cutting edge: MyD88 controls phagocyte NADPH oxidase function and killing of gram-negative bacteria. J. Immunol. 175, 5596-5600.

Lee, R. J., Liu, C. W., Harty, C., Mccracken, A. A., Latterich, M., Romisch, K., Demartino, G. N., Thomas, P. J., and Brodsky, J. L. (2004). Uncoupling retrotranslocation and degradation in the ER-associated degradation of a soluble protein. EMBO J. 23, 2206-2215.

Lelouard, H., Ferrand, V., Marguet, D., Bania, J., Camosseto, V., David, A. Gatti, E., and Pierre, P. (2004). Dendritic cell aggresome-like induced structures are dedicated areas for ubiquitination and storage of newly synthesized defective proteins. J. Cell Biol. 164, 667-675.

Lennon-Dumenil, A. M., Bakker, A. H., Maehr, R., Fiebiger, E., Overkleeft, H. S., Rosemblatt, M., Ploegh, H. L., and Lagaudriere-Gesbert, C. (2002). Analysis of protease activity in live antigen-presenting cells shows regulation of the phagosomal proteolytic contents during dendritic cell activation. J. Exp. Med. 196, 529-540.

Li, G., Zhou, X., Zhao, G., Schindelin, H., and Lennarz, W. J. (2005). Multiple modes of interaction of the deglycosylation enzyme, mouse peptide $N$-glycanase, with the proteasome. Proc. Natl. Acad. Sci. U.S.A. 102, 15809-15814.

Lin, M. L., Zhan, Y., Proietto, A. I., Prato, S., Wu, L., Heath, W. R., Villadangos, J. A., and Lew, A. M. (2008). Selective suicide of cross-presenting CD8+ dendritic cells by cytochrome $c$ injection shows functional heterogeneity within this subset. Proc. Natl. Acad. Sci. U.S.A. 105, 3029-3034.

Lipson, C., Alalouf, G., Bajorek, M., Rabinovich, E., Atir-Lande, A., Glickman, M., and Bar-Nun, S. (2008). A proteasomal ATPase contributes to dislocation of endoplasmic reticulum-associated degradation (ERAD) substrates. J. Biol. Chem. 283, 7166-7175.

Lutz, M. B., Rovere, P., Kleijmeer, M. J., Rescigno, M., Assmann, C. U., Oorschot, V. M., Geuze, H. J., Trucy, J., Demandolx, D., Davoust, J., and Ricciardi-Castagnoli, P. (1997). Intracellular routes and selective retention of antigens in mildly acidic cathepsin D/lysosome-associated membrane protein-1/MHC class II-positive vesicles in immature dendritic cells. J. Immunol. 159, 3707-3716.

Machy, P., Serre, K., and Leserman, L. (2000). Class I-restricted presentation of exogenous antigen acquired by Fcgamma receptor-mediated endocytosis is regulated in dendritic cells. Eur. J. Immunol. 30, 848-857. 
Mayer, T. U., Braun, T., and Jentsch, S. (1998). Role of the proteasome in membrane extraction of a short-lived ER-transmembrane protein. EMBO J. 17, 3251-3257.

Merzougui, N., Kratzer, R., Saveanu, L., and Van Endert, P. (2011). A proteasome-dependent, TAP-independent pathway for cross-presentation of phagocytosed antigen. EMBO Rep. 12, 1257-1264.

Molinari, M., Galli, C., Piccaluga, V., Pieren, M., and Paganetti, P. (2002). Sequential assistance of molecular chaperones and transient formation of covalent complexes during protein degradation from the ER. J. Cell Biol. 158, 247-257.

Morreale, G., Conforti, L., Coadwell, J., Wilbrey, A. L., and Coleman, M. P. (2009). Evolutionary divergence of valosin-containing protein/cell division cycle protein 48 binding interactions among endoplasmic reticulum-associated degradation proteins. FEBS J. 276, 1208-1220.

Muller-Taubenberger, A., Lupas, A. N., Li, H., Ecke, M., Simmeth, E., and Gerisch, G. (2001). Calreticulin and calnexin in the endoplasmic reticulum are important for phagocytosis. EMBO J. 20, 6772-6782.

Pfeifer, J. D., Wick, M. J., Roberts, R. L., Findlay, K., Normark, S. J., and Harding, C. V. (1993). Phagocytic processing of bacterial antigens for class I MHC presentation to T cells. Nature 361, 359-362.

Platt, C. D., Ma, J. K., Chalouni, C., Ebersold, M., Bou-Reslan, H., Carano, R. A., Mellman, I., and Delamarre, L. (2010). Mature dendritic cells use endocytic receptors to capture and present antigens. Proc. Natl. Acad. Sci. U.S.A. 107, 4287-4292.

Rabinovich, E., Kerem, A., Frohlich, K. U., Diamant, N., and Bar-Nun, S. (2002). AAA-ATPase p97/Cdc48p, a cytosolic chaperone required for endoplasmic reticulum-associated protein degradation. Mol. Cell. Biol. 22, 626-634.

Regnault, A., Lankar, D., Lacabanne, V., Rodriguez, A., Thery, C., Rescigno, M., Saito, T., Verbeek, S., Bonnerot, C., Ricciardi-Castagnoli, P., and Amigorena, S. (1999a). Fcgamma receptor-mediated induction of dendritic cell maturation and major histocompatibility complex class Irestricted antigen presentation after immune complex internalization. J. Exp. Med. 189, 371-380.

Rescigno, M., Citterio, S., Thery, C., Rittig, M., Medaglini, D., Pozzi, G., Amigorena, S., and Ricciardi-Castagnoli, P. (1998).
Bacteria-induced neo-biosynthesis, stabilization, and surface expression of functional class I molecules in mouse dendritic cells. Proc. Natl. Acad. Sci. U.S.A. 95, 5229-5234.

Russell, D. G., and Yates, R. M. (2007). Toll-like receptors and phagosome maturation. Nat. Immunol. 8, 217; Author reply 217-218.

Sancho, D., Joffre, O. P., Keller, A. M., Rogers, N. C., Martinez, D., Hernanz-Falcon, P., Rosewell, I., and Reis e Sousa, C. (2009). Identification of a dendritic cell receptor that couples sensing of necrosis to immunity. Nature 458, 899-903.

Sancho, D., Mourao-Sa, D., Joffre, O. P., Schulz, O., Rogers, N. C., Pennington, D. J., Carlyle, J. R., and Reis e Sousa, C. (2008). Tumor therapy in mice via antigen targeting to a novel, DC-restricted C-type lectin. J. Clin. Invest. 118, 2098-2110.

Sanjuan, M. A., Dillon, C. P., Tait, S. W., Moshiach, S., Dorsey, F., Connell, S., Komatsu, M., Tanaka, K., Cleveland, J. L., Withoff, S., and Green, D. R. (2007). Toll-like receptor signalling in macrophages links the autophagy pathway to phagocytosis. Nature 450 , 1253-1257.

Savina, A., Jancic, C., Hugues, S., Guermonprez, P., Vargas, P., Moura, I. C., Lennon-Dumenil, A. M. Seabra, M. C., Raposo, G., and Amigorena, S. (2006). NOX2 controls phagosomal $\mathrm{pH}$ to regulate antigen processing during crosspresentation by dendritic cells. Cell 126 , 205-218.

Savina, A., Peres, A., Cebrian, I., Carmo, N., Moita, C., Hacohen, N., Moita, L. F., and Amigorena, S. (2009). The small GTPase Rac2 controls phagosomal alkalinization and antigen crosspresentation selectively in $\mathrm{CD} 8(+)$ dendritic cells. Immunity 30, 544-555.

Schnorrer, P., Behrens, G. M., Wilson, N. S., Pooley, J. L., Smith, C. M., ElSukkari, D., Davey, G., Kupresanin, F., Li, M., Maraskovsky, E., Belz, G. T., Carbone, F. R., Shortman, K., Heath, W. R., and Villadangos, J. A. (2006). The dominant role of CD8+ dendritic cells in cross-presentation is not dictated by antigen capture. Proc. Natl. Acad. Sci. U.S.A. 103 , 10729-10734.

Schulze, A., Standera, S., Buerger, E., Kikkert, M., Van Voorden, S., Wiertz, E., Koning, F., Kloetzel, P. M., and Seeger, M. (2005). The ubiquitindomain protein HERP forms a complex with components of the endoplasmic reticulum associated degradation pathway. J. Mol. Biol. 354, 1021-1027.
Shakushiro, K., Yamasaki, Y., Nishikawa, M., and Takakura, Y. (2004). Efficient scavenger receptor-mediated uptake and cross-presentation of negatively charged soluble antigens by dendritic cells. Immunology 112, 211-218.

Shen, L., Sigal, L. J., Boes, M., and Rock, K. L. (2004). Important role of cathepsin $\mathrm{S}$ in generating peptides for TAP-independent MHC class I crosspresentation in vivo. Immunity 21, 155-165.

Singh, R., and Cresswell, P. (2010). Defective cross-presentation of viral antigens in GILT-free mice. Science 328, 1394-1398.

Swanson, J. A., and Hoppe, A. D. (2004). The coordination of signaling during Fc receptor-mediated phagocytosis. J. Leukoc. Biol. 76, 1093-1103.

Tacken, P. J., De Vries, I. J., Gijzen, K., Joosten, B., Wu, D., Rother, R. P., Faas, S. J., Punt, C. J., Torensma, R., Adema, G. J., and Figdor, C. G. (2005). Effective induction of naive and recall $\mathrm{T}$-cell responses by targeting antigen to human dendritic cells via a humanized anti-DC-SIGN antibody. Blood 106, 1278-1285.

Taylor, M., Navarro-Garcia, F., Huerta, J., Burress, H., Massey, S., Ireton, K., and Teter, K. (2010). Hsp90 is required for transfer of the cholera toxin Al subunit from the endoplasmic reticulum to the cytosol. J. Biol. Chem. 285, 31261-31267.

Trombetta, E. S., Ebersold, M., Garrett, W., Pypaert, M., and Mellman, I. (2003). Activation of lysosomal function during dendritic cell maturation. Science 299, 1400-1403.

Ushioda, R., Hoseki, J., Araki, K., Jansen, G., Thomas, D. Y., and Nagata, K. (2008). ERdj5 is required as a disulfide reductase for degradation of misfolded proteins in the ER. Science 321, 569-572.

van Montfoort, N., Camps, M. G., Khan, S., Filippov, D. V., Weterings, J. J., Griffith, J. M., Geuze, H. J., Van Hall, T., Verbeek, J. S., Melief, C. J., and Ossendorp, F. (2009). Antigen storage compartments in mature dendritic cells facilitate prolonged cytotoxic $\mathrm{T}$ lymphocyte cross-priming capacity. Proc. Natl. Acad. Sci. U.S.A. 106, 6730-6735.

Vembar, S. S., and Brodsky, J. L. (2008). One step at a time: endoplasmic reticulum-associated degradation. Nat. Rev. Mol. Cell Biol. 9, 944-957.

Villadangos, J. A., Schnorrer, P., and Wilson, N. S. (2005). Control of MHC class II antigen presentation in dendritic cells: a balance between creative and destructive forces. Immunol. Rev. 207, 191-205.
Villadangos, J. A., and Shortman, K. (2010). Found in translation: the human equivalent of mouse CD8+ dendritic cells. J. Exp. Med. 207, 1131-1134.

Vulcano, M., Dusi, S., Lissandrini, D., Badolato, R., Mazzi, P., Riboldi, E., Borroni, E., Calleri, A., Donini, M., Plebani, A., Notarangelo, L., Musso, T., and Sozzani, S. (2004). Toll receptor-mediated regulation of NADPH oxidase in human dendritic cells. J. Immunol. 173, 5749-5756.

Wagner, C. S., and Cresswell, P. (2012). TLR and nucleotide-binding oligomerization domain-like receptor signals differentially regulate exogenous antigen presentation. $J$. Immunol. 188, 686-693.

Weck, M. M., Grunebach, F., Werth, D., Sinzger, C., Bringmann, A., and Brossart, P. (2007). TLR ligands differentially affect uptake and presentation of cellular antigens. Blood 109, 3890-3894.

West, M. A., Wallin, R. P., Matthews, S. P., Svensson, H. G., Zaru, R., Ljunggren, H. G., Prescott, A. R., and Watts, C. (2004). Enhanced dendritic cell antigen capture via toll-like receptor-induced actin remodeling. Science 305, 1153-1157.

Wilson, N. S., Behrens, G. M., Lundie, R. J., Smith, C. M., Waithman, J., Young, L., Forehan, S. P., Mount, A., Steptoe, R. J., Shortman, K. D., De Koning-Ward, T. F., Belz, G. T., Carbone, F. R., Crabb, B. S., Heath, W. R., and Villadangos, J. A. (2006). Systemic activation of dendritic cells by Toll-like receptor ligands or malaria infection impairs crosspresentation and antiviral immunity. Nat. Immunol. 7, 165-172.

Wilson, N. S., and Villadangos, J. A. (2005). Regulation of antigen presentation and cross-presentation in the dendritic cell network: facts, hypothesis, and immunological implications. Adv. Immunol. 86, 241-305.

Yates, R. M., Hermetter, A., and Russell, D. G. (2005). The kinetics of phagosome maturation as a function of phagosome/lysosome fusion and acquisition of hydrolytic activity. Traffic 6, 413-420.

Yates, R. M., and Russell, D. G. (2005). Phagosome maturation proceeds independently of stimulation of tolllike receptors 2 and 4. Immunity 23, 409-417.

Ye, Y., Meyer, H. H., and Rapoport, T. A. (2001). The AAA ATPase Cdc48/p97 and its partners transport proteins from the ER into the cytosol. Nature 414, 652-656.

Ye, Y., Shibata, Y., Kikkert, M., Van Voorden, S., Wiertz, E., and Rapoport, T. 
A. (2005). Recruitment of the p97 ATPase and ubiquitin ligases to the site of retrotranslocation at the endoplasmic reticulum membrane. Proc. Natl. Acad. Sci. U.S.A. 102, 14132 14138.

Zehner, M., Chasan, A. I., Schuette, V., Embgenbroich, M., Quast, T., Kolanus, W., and Burgdorf, S. (2011). Mannose receptor polyubiquitination regulates endosomal recruitment of p97 and cytosolic antigen translocation for cross-presentation.
Proc. Natl. Acad. Sci. U.S.A. 108, 9933-9938.

Zhong, X., Shen, Y., Ballar, P., Apostolou, A., Agami, R., and Fang, S. (2004). AAA ATPase p97/valosincontaining protein interacts with gp78, a ubiquitin ligase for endoplasmic reticulum-associated degradation. J. Biol. Chem. 279, 4567645684.

Conflict of Interest Statement: The authors declare that the research was conducted in the absence of any commercial or financial relationships that could be construed as a potential conflict of interest.

Received: 27 March 2012; accepted: 14 May 2012; published online: 04 June 2012.

Citation: Wagner CS, Grotzke JE and Cresswell P (2012) Intracellular events regulating cross-presentation. Front. Immun. 3:138. doi: 10.3389/fimmu. 2012.00138
This article was submitted to Frontiers in Antigen Presenting Cell Biology, a specialty of Frontiers in Immunology.

Copyright (c) 2012 Wagner, Grotzke and Cresswell. This is an open-acces article distributed under the terms of the Creative Commons Attribution Non Commercial License, which permits non-commercial use, distribution, and reproduction in other forums, provided the original authors and source are credited. 